\title{
Can the Resonance Raman Optical Activity Spectrum Display Sign Alternation?
}

\author{
Luciano N. Vidal, ${ }^{\dagger}$ Tommaso Giovannini, ${ }^{\ddagger}$ and Chiara Cappelli*, \\ Universidade Tecnológica Federal do Paraná, Rua Dep. Heitor de Alencar Furtado, 4900, \\ 81280-340, Curitiba, Brazil, and Scuola Normale Superiore, Piazza dei Cavalieri 7, 56126 \\ Pisa, Italy \\ E-mail: chiara.cappelli@sns.it
}

\section{S1 The SES-FC Mechanism for RROA}

In the case of backscattering unpolarized Incident Circular Polarization (ICP) ROA, the intensity of a vibrational transition in RROA and its corresponding intensity in RR are related in the following way: ${ }^{1}$

$$
I_{\mathrm{RROA}}^{\mathrm{ICP}}=-\frac{1}{2} g_{r g} I_{\mathrm{RR}}^{\mathrm{ICP}}
$$

$g_{r g}$ is the so-called anisotropy ratio, i.e. the ratio of electronic circular dichroism (ECD) intensity to the corresponding UV-vis absorption intensity for the transition from the ground electronic state $g$ to the resonance state $r$. Therefore, the sign of all RROA bands are equal and opposite in sign to the ECD band corresponding to the laser excitation frequency $\omega_{0}$

\footnotetext{
${ }^{*}$ To whom correspondence should be addressed

${ }^{\dagger}$ Universidade Tecnológica Federal do Paraná, Rua Dep. Heitor de Alencar Furtado, 4900, 81280-340, Curitiba, Brazil

${ }^{\ddagger}$ Scuola Normale Superiore, Piazza dei Cavalieri 7, 56126 Pisa, Italy
} 
used to measure the RR spectrum. ${ }^{2}$ Relation S1 can be demonstrated if the molecule fixed coordinate system is chosen such that the electric dipole electronic transition moment lies along one of the coordinate axes. ${ }^{1,3}$ In this particular orientation, the RR polarizability tensor for a given $f \leftarrow i$ vibrational transition, $\boldsymbol{\alpha}^{f i}$, has only one non-zero component, say $\alpha_{z z}^{f i}$, the two non-vanishing RR rotational invariants are proportional to the square of $\alpha_{z z}^{f i}$ and the RR intensity, for a fixed laser excitation frequency and temperature, is proportional to $\left|\alpha_{z z}^{f i}\right|^{2}$ as well.

At the most general level of theory, the ROA intensities are given in terms of ten rotational invariants involving products between $\boldsymbol{\alpha}^{f i}$ and one of the four ROA tensors. ${ }^{3,4}$ Within the SES limit, there is no contribution from the electric dipole-electric quadrupole tensors $\mathbf{A}^{f i}$ and $\mathcal{A}^{f i}$ to the RROA scattering while the four non-vanishing rotational invariants of the electric dipole-magnetic dipole tensors $\mathbf{G}^{f i}$ and $\mathcal{G}^{f i}$ and the RROA intensities are all proportional to $\Im\left(\alpha_{z z}^{f i} G_{z z}^{f i *}\right)$. Consequently, the ratio between the RR and RROA intensities of the same vibrational transition gives equation S1 for the backscattering unpolarized ICP ROA experiment. As already mentioned above, this theoretical framework is that commonly exploited to treat RROA. 


\section{S2 Theory of RROA Vibronic Polarizabilities}

Within the general unrestricted theory of ROA, ${ }^{3}$ the ROA intensities are given in terms of ten rotational invariants which are built from products between the electric dipole-electric dipole Raman polarizability, $\boldsymbol{\alpha}$, and one of the four ROA tensors: (i) $\boldsymbol{G}$, the electric dipole-magnetic dipole polarizability tensor, (ii) $\mathcal{G}$, the magnetic dipole-electric dipole tensor, (iii) $\boldsymbol{A}$, the electric dipole-electric quadrupole tensor and (iv) $\mathcal{A}$, the electric quadrupole-electric dipole tensor. In order to evaluate Raman and ROA tensors, the electronic transition moments must be written as a function of vibrational coordinates. This can be achieved by expanding them in a Taylor series of, for instance, the normal coordinates of the ground electronic state: ${ }^{5,6}$

$$
\left\langle e_{g}\left|\hat{o}_{a}\right| e_{r}\right\rangle=o_{a, 0}^{g r}+\sum_{k} o_{a, k}^{g r} Q_{k}^{g}+\frac{1}{2} \sum_{k} \sum_{l} o_{a, k l}^{g r} Q_{k}^{g} Q_{l}^{g}+\cdots
$$

where $\left\langle e_{g}\left|\hat{o}_{a}\right| e_{r}\right\rangle$ is a transition moment between the ground, $e_{g}$, and excited state $e_{r}$, of the operator $\hat{o}_{a}$, which represents a Cartesian component of the electric dipole, magnetic dipole or electric quadrupole operators. In Equation (S2), $o_{a, 0}^{g r}, o_{a, k}^{g r}$ and $o_{a, k l}^{g r}$ correspond to $\left\langle e_{g}\left|\hat{o}_{a}\right| e_{r}\right\rangle$ and its first and second derivatives, respectively, evaluated at the equilibrium ground state geometry. At the Franck-Condon (FC) level, expansion (S2) is truncated at the zeroth order term $o_{a, 0}^{g r}$, giving rise to the following general expression for the Raman/ROA tensors:

$$
T_{a b}^{\mathrm{FC}}=\frac{1}{\hbar} \sum_{\left\{e_{r}\right\}} \sum_{n} \frac{o_{a, 0}^{g r} o_{b, 0}^{r g}\left\langle f^{g} \mid n^{r}\right\rangle\left\langle n^{r} \mid i^{g}\right\rangle}{\omega_{r g}+\omega_{n i}-\omega_{0}-i \Gamma_{e_{r}}}
$$

An extension to the FC principle was proposed by Herzberg and Teller: ${ }^{7}$ in this case, it accounts for a linear variation of the transition moment with respect to the normal coordinates, i.e., the first order terms $o_{a, k}^{g r}$ of Equation (S2) are considered. At the Herzberg-Teller (HT) level, any of the five Raman/ROA tensors related to the resonance scattering have the 
general form given below:

$$
\begin{aligned}
T_{a b}= & T_{a b}^{\mathrm{FC}}+\sum_{\left\{e_{r}\right\}}\left(\sum_{n, k} \frac{o_{a, k}^{g r} o_{b, 0}^{r g}\left\langle f^{g}\left|Q_{k}^{g}\right| n^{r}\right\rangle\left\langle n^{r} \mid i^{g}\right\rangle}{\omega_{r g}+\omega_{n i}-\omega_{0}-i \Gamma_{e_{r}}}\right. \\
& \left.+\sum_{n, k} \frac{o_{a, 0}^{g r} r_{b, k}^{r g}\left\langle f^{g} \mid n^{r}\right\rangle\left\langle n^{r}\left|Q_{k}^{g}\right| i^{g}\right\rangle}{\omega_{r g}+\omega_{n i}-\omega_{0}-i \Gamma_{e_{r}}}+\sum_{n, k, l} \frac{o_{a, k}^{g r} o_{b, l}^{r g}\left\langle f^{g}\left|Q_{k}^{g}\right| n^{r}\right\rangle\left\langle n^{r}\left|Q_{l}^{g}\right| i^{g}\right\rangle}{\omega_{r g}+\omega_{n i}-\omega_{0}-i \Gamma_{e_{r}}}\right)
\end{aligned}
$$

where $\left|i^{g}\right\rangle$ and $\left|f^{g}\right\rangle$ are the initial and final vibrational wavefunctions of the ground $e_{g}$ and $\left|n^{r}\right\rangle$ is an intermediate vibrational wavefunction of the excited state $e_{r}$. The denominators

$\omega_{r g}=\left(E_{e_{r}}-E_{e_{g}}\right) / \hbar$ and $\omega_{n i}=\left(E_{n^{r}}-E_{i^{g}}\right) / \hbar$ are energy differences between electronic and vibrational states, respectively. The $\Gamma_{e_{r}}$ term is related to the lifetime of the excited state $e_{r}$ and is assumed to be independent of the vibrational state $n^{r}$. The electronic states between brackets in Equations (S3) and (S4), $\left\{e_{r}\right\}$, are those in resonance with the incident radiation while $n$ represents an intermediate vibrational state.

\section{S3 Computational details}

The computational procedure adopted in this work is the same reported in reference 6 , including those approximations necessary to remove the origin dependence from the FC and HT RROA cross sections. The electronic structure calculations were performed with a development version of the Gaussian suite of programs. ${ }^{8}$ Ground and excited state properties were computed using the Density Functional Theory (DFT) and its time-dependent extension (TD-DFT) with the B3LYP ${ }^{9}$ functional and the polarized double- $\zeta$ SNSD basis set. ${ }^{10,11}$ All Raman and ROA tensors were calculated using the velocity representation of the electric dipole and traceless electric quadrupole transition moments. ${ }^{12}$ All vibronic polarizabilities were computed in a sum-over-state fashion, and the FC and HT integrals in Equations (S3) and (S4) were evaluated by describing the potential energy surface (PES) of both ground and excited electronic states within the harmonic approximation. The data required to evaluate FC and HT integrals were obtained from the ground state geometry and its respective 
analytic Hessian but for the excited state properties two different approaches were used: ${ }^{5}$ The Adiabatic Hessian (AH) model, where both PESs are treated at the same level, therefore an excited state geometry optimization and harmonic frequencies calculation is performed and the Vertical Gradient (VG) model, where the excited state Hessian is assumed to be equal to the ground state one. The FC and HT integrals as well as the five types of polarizabilities required to obtain the resonance ROA spectrum were computed by extending to RROA the code developed by some of the present authors for Resonance Raman and described in Ref. ${ }^{13}$ The ROA intensities reported in the current work are those from the backscattering SCP experiment with the sample being illuminated with unpolarized light.

All results consider fully isotropically averaged samples.

\section{S4 (1R)-Camphorquinone}

Table S1: B3LYP/SNS-D/PCM Vertical Excitation Wavelengths $(\lambda)$, in nm), Oscillator Strengths $(f)$, and Rotatory Strengths $\left(R\right.$,in $10^{-40}$ erg esu cm/gauss ) for (1R)Camphorquinone in cyclohexane. The highlighted states are the ones considered in the VG calculations.

\begin{tabular}{cccc}
\hline State & $\lambda$ & $f$ & $R$ \\
\hline S1 & 499 & 0.0003 & -0.73 \\
S2 & 295 & 0.0002 & 1.28 \\
S3 & 237 & 0.0063 & -9.63 \\
S4 & 222 & 0.0031 & -5.44 \\
S5 & $\mathbf{2 1 4}$ & $\mathbf{0 . 0 1 4 4}$ & $\mathbf{5 3 . 4 1}$ \\
S6 & $\mathbf{2 1 1}$ & $\mathbf{0 . 0 1 9 4}$ & $\mathbf{- 4 6 . 7 5}$ \\
S7 & 205 & 0.0099 & -11.52 \\
S8 & 205 & 0.0004 & -0.90 \\
S9 & 204 & 0.0117 & 14.30 \\
S10 & 200 & 0.0141 & -0.48 \\
\hline
\end{tabular}




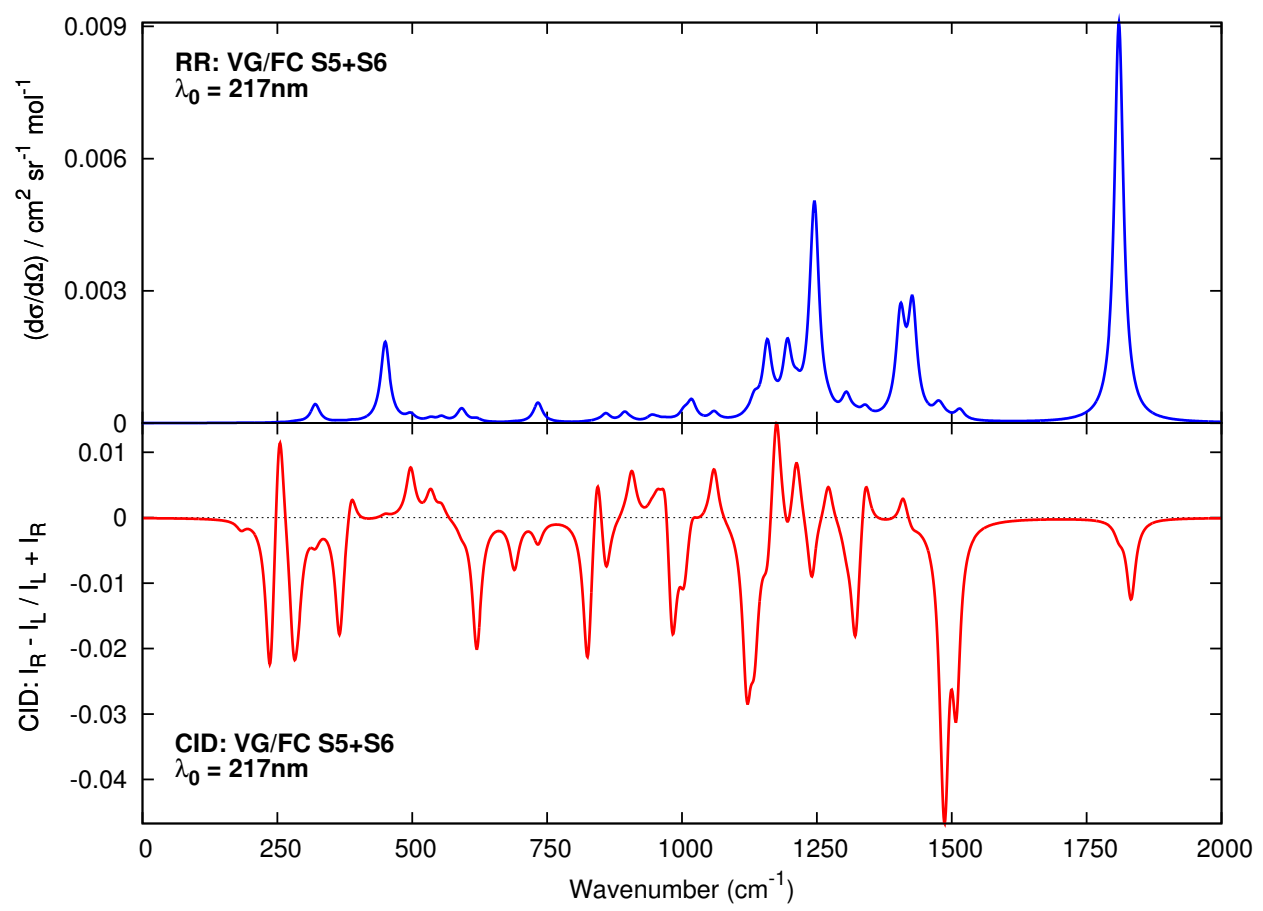

Figure S1: B3LYP/SNS-D/PCM calculated Resonance Raman (top) and Circular Intensity Difference (CID) (bottom) spectra (1R)-Camphorquinone in cyclohexane solution. Excitation energy $\lambda_{0}=217 \mathrm{~nm}$.

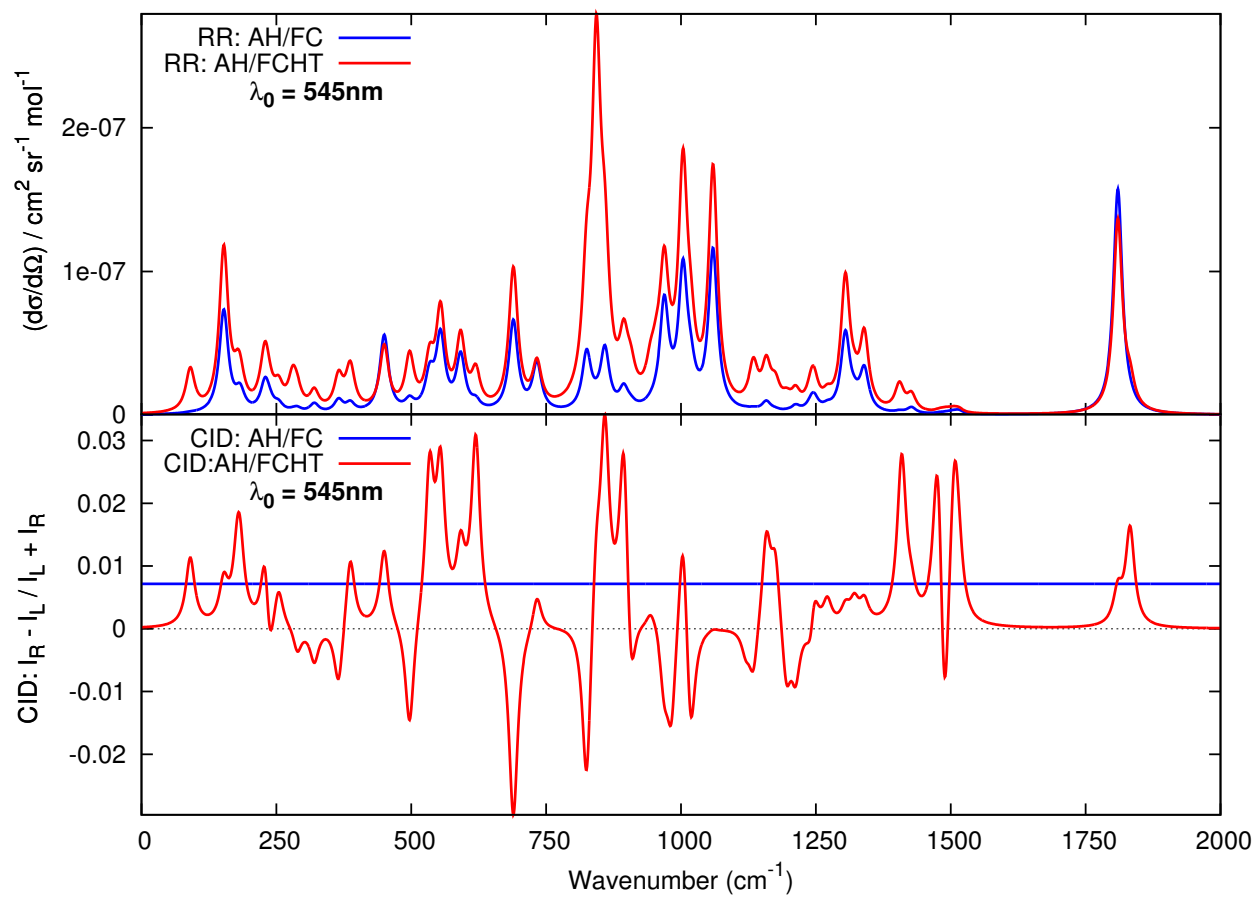

Figure S2: B3LYP/SNS-D/PCM calculated Resonance Raman (top) and Circular Intensity Difference (CID) (bottom) spectra (1R)-Camphorquinone in cyclohexane solution. Excitation energy $\lambda_{0}=545 \mathrm{~nm}$. 


\section{S5 (S)-Nicotine}

Table S2: CAM-B3LYP/aug-cc-pVDZ/PCM Vertical Excitation Wavelengths $(\lambda)$, in nm), Oscillator Strengths $(f)$, and Rotatory Strengths $\left(R\right.$,in $10^{-40}$ erg esu cm/gauss) of the two conformers of (S)-nicotine as reported in ref. ${ }^{14}$ Highlighted states are the ones considered in the VG calculations.

\begin{tabular}{c|ccc|ccc}
\hline State & \multicolumn{3}{|c|}{ Nicotine A } & \multicolumn{3}{c}{ Nicotine B } \\
& $\lambda$ & $f$ & $R$ & $\lambda$ & $f$ & $R$ \\
\hline S1 & $\mathbf{2 4 0}$ & $\mathbf{0 . 0 0 5 6}$ & $\mathbf{- 1 5 . 0 9}$ & $\mathbf{2 4 2}$ & $\mathbf{0 . 0 0 8 1}$ & $\mathbf{- 2 3 . 8 3}$ \\
S2 & $\mathbf{2 3 1}$ & $\mathbf{0 . 0 2 6 4}$ & $\mathbf{1 . 6 4}$ & $\mathbf{2 2 9}$ & $\mathbf{0 . 0 7 0 8}$ & $\mathbf{3 4 . 6 4}$ \\
S3 & $\mathbf{2 2 8}$ & $\mathbf{0 . 0 5 7 7}$ & $\mathbf{1 0 . 7 4}$ & $\mathbf{2 2 8}$ & $\mathbf{0 . 0 0 6 9}$ & $\mathbf{3 . 6 1}$ \\
S4 & 218 & 0.0004 & 0.41 & 217 & 0.0042 & -12.33 \\
S5 & 212 & 0.0343 & -22.03 & 212 & 0.0031 & -1.70 \\
S6 & 209 & 0.0113 & 6.96 & 211 & 0.0222 & -6.46 \\
S7 & 200 & 0.0704 & -7.06 & 201 & 0.0650 & 15.52 \\
S8 & 197 & 0.0844 & 0.13 & 198 & 0.1113 & -12.87 \\
S9 & 193 & 0.0170 & -5.36 & 194 & 0.0211 & -4.20 \\
S10 & 188 & 0.0084 & 5.99 & 190 & 0.0161 & -24.53 \\
S11 & 184 & 0.0095 & -24.09 & 185 & 0.0123 & 21.89 \\
S12 & 179 & 0.2363 & 39.71 & 180 & 0.1738 & -10.80 \\
\hline
\end{tabular}

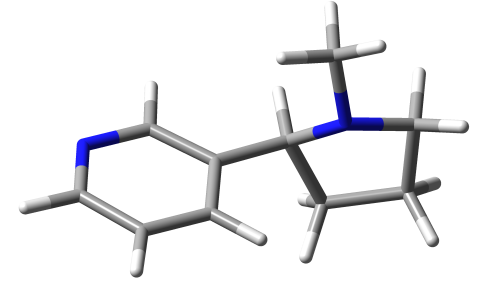

(a)

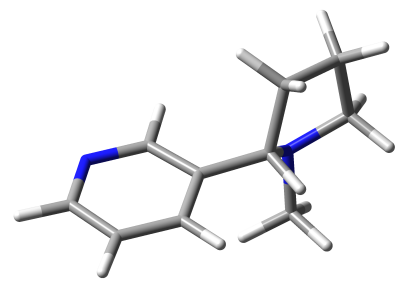

(b)

Figure S3: Nicotine molecule in aqueous solution described by means of PCM. (a) Conformer A - Boltzmann population 55.3\%; (b) Confermer B - Boltzmann population $44.7 \%$. 


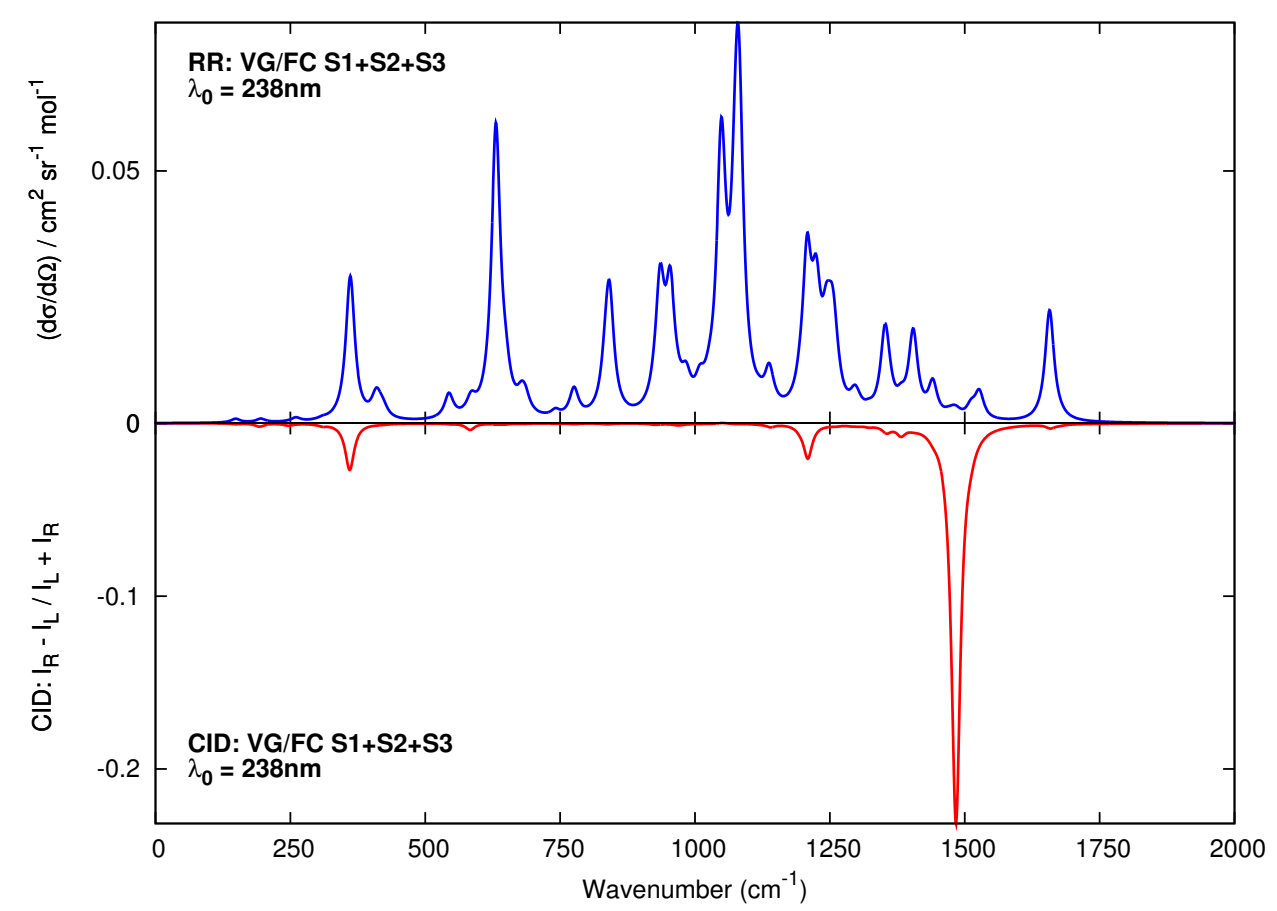

Figure S4: B3LYP/SNS-D/PCM calculated Resonance Raman (top) and Circular Intensity Difference (CID) (bottom) spectra (S)-Nicotine in aqueous solution. Excitation energy $\lambda_{0}=238 \mathrm{~nm}$.

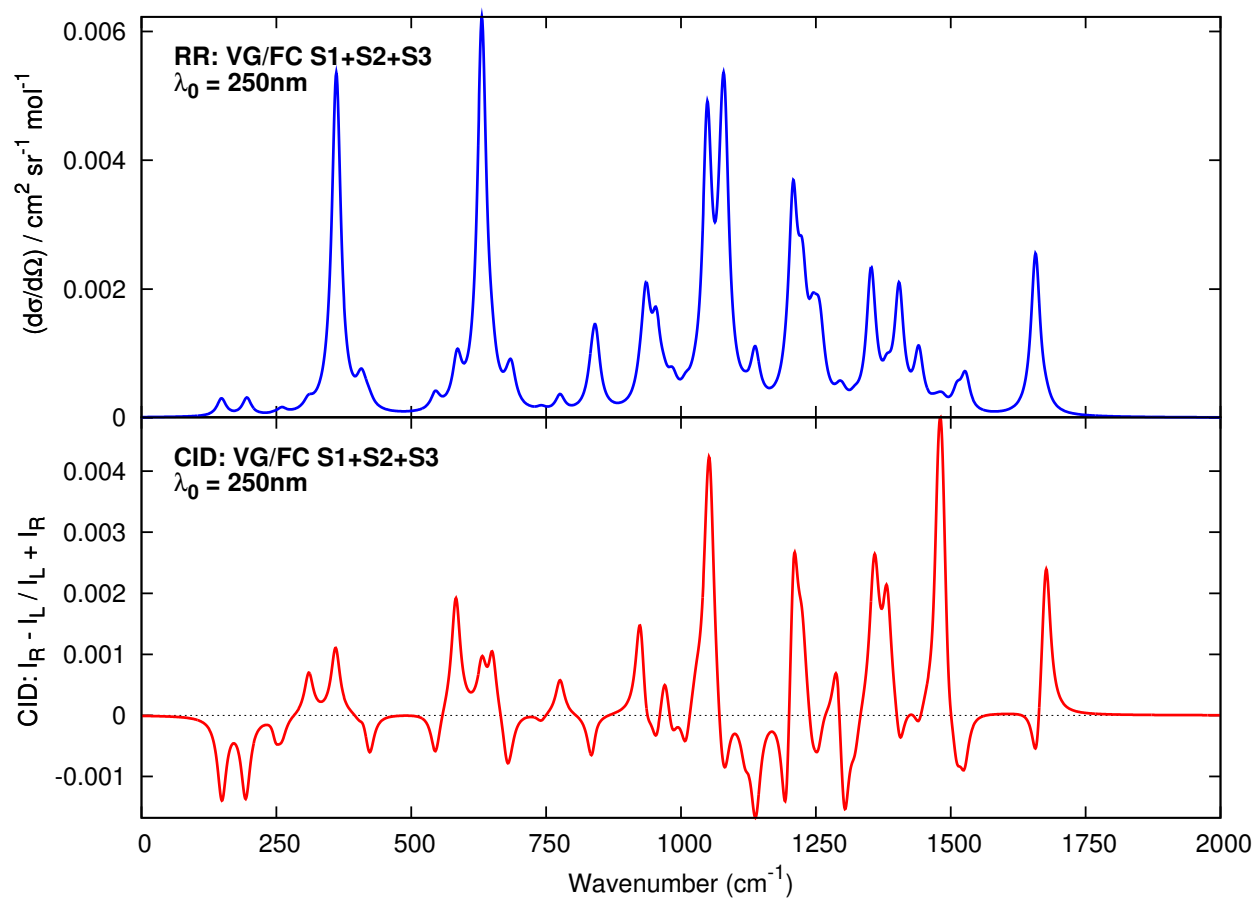

Figure S5: B3LYP/SNS-D/PCM calculated Resonance Raman (top) and Circular Intensity Difference (CID) (bottom) spectra (S)-Nicotine in aqueous solution. Excitation energy $\lambda_{0}=250 \mathrm{~nm}$. 


\section{References}

(1) Nafie, L. A. Theory of Resonance Raman Optical Activity: the Single Electronic State Limit. Chem. Phys. 1996, 205, 309-322.

(2) Vargek, M.; Freedman, T. B.; Lee, E.; Nafie, L. A. Experimental Observation of Resonance Raman Optical Activity. Chem. Phys. Lett. 1998, 287, 359-364.

(3) Nafie, L. A. Vibrational Optical Activity; John Wiley \& Sons: Chichester, 2011.

(4) Hecht, L.; Nafie, L. A. Theory of natural Raman Optical Activity Part I. Complete Circular Polarization Formalism. Mol. Phys. 1991, 72, 441-469.

(5) Barone, V., Ed. Computational Strategies for Spectroscopy; John Wiley \& Sons: New Jersey, 2012.

(6) Vidal, L. N.; Egidi, F.; Barone, V.; Cappelli, C. Origin Invariance in Vibrational Resonance Raman Optical Activity. J. Chem. Phys. 2015, 142, 174101.

(7) Herzberg, G.; Teller, E. Schwingungsstruktur der Elektronenübergänge bei mehratomigen Molekülen Z. Phys. Chem. B Chem. E 1933, 21, 410.

(8) Frisch, M. J.; Trucks, G. W.; Schlegel, H. B.; Scuseria, G. E.; Robb, M. A.; Cheeseman, J. R.; Scalmani, G.; Barone, V.; Mennucci, B.; Petersson, G. A.; Nakatsuji, H.; Caricato, M.; Li, X.; Hratchian, H. P.; Izmaylov, A. F.; Bloino, J.; Janesko, B. G.; Lipparini, F.; Zheng, G.; Sonnenberg, J. L.; Liang, W.; Hada, M.; Ehara, M.; Toyota, K.; Fukuda, R.; Hasegawa, J.; Ishida, M.; Nakajima, T.; Honda, Y.; Kitao, O.; Nakai, H.; Vreven, T.; Montgomery, J. A.; Jr.,; Peralta, J. E.; Ogliaro, F.; Bearpark, M.; Heyd, J. J.; Brothers, E.; Kudin, K. N.; Staroverov, V. N.; Keith, T.; Kobayashi, R.; Normand, J.; Raghavachari, K.; Rendell, A.; Burant, J. C.; Iyengar, S. S.; Tomasi, J.; Cossi, M.; Rega, N.; Millam, J. M.; Klene, M.; Knox, J. E.; Cross, J. B.; Bakken, V.; Adamo, C.; Jaramillo, J.; Gomperts, R.; Stratmann, R. E.; 
Yazyev, O.; Austin, A. J.; Cammi, R.; Pomelli, C.; Ochterski, J. W.; Martin, R. L.; Morokuma, K.; Zakrzewski, V. G.; Voth, G. A.; Salvador, P.; Dannenberg, J. J.; Dapprich, S.; Parandekar, P. V.; Mayhall, N. J.; Daniels, A. D.; Farkas, O.; Foresman, J. B.; Ortiz, J. V.; Cioslowski, J.; Fox, D. J. Gaussian Development Version, Revision H.37+. Gaussian, Inc., Wallingford CT, 2010.

(9) Becke, A. D. Density-functional Thermochemistry. III. The Role of Exact Exchange. J. Chem. Phys. 1993, 98, 5648-5652.

(10) Barone, V.; Biczysko, M.; Bloino, J. Fully Anharmonic IR and Raman Spectra of Medium-size Molecular Systems: Accuracy and Interpretation. Phys. Chem. Chem. Phys. 2014, 16, 1759-1787.

(11) Double and triple-zeta basis sets of SNS families are available for download, visit http://dreamsnet.sns.it/downloads (accessed August 25, 2016).

(12) Luber, S.; Reiher, M. Raman Optical Activity Spectra of Chiral Transition Metal Complexes. Chem. Phys. 2008, 346, 212-223.

(13) Egidi, F.; Bloino, J.; Cappelli, C.; Barone, V. A Robust and Effective Time-independent Route to the Calculation of Resonance Raman Spectra of Large Molecules in Condensed Phases with the Inclusion of Duschinsky, Herzberg-Teller, Anharmonic, and Environmental Effects. J. Chem. Theory Comput. 2014, 10, 346-363.

(14) Egidi, F.; Russo, R.; Carnimeo, I.; D’Urso, A.; Mancini, G.; Cappelli, C. The Electronic Circular Dichroism of Nicotine in Aqueous Solution: A Test Case for Continuum and Mixed Explicit-Continuum Solvation Approaches. The Journal of Physical Chemistry A 2015, 119, 5396-5404. 